\title{
SUR LES PROTEINES \\ DU PLASMA SÉMINAL DU CHEVAL
}

PAR

\author{
P. SZUMOWSKI et M. STOEBER \\ Laboratoire de recherches de la Chaire de Zootechnie, \\ École nationale vétérinaire, Alfort
}

\section{INTRODUCTION}

Le sperme de cheval, par comparaison avec celui d'autres espèces, humaine ou bovine par exemple, est très peu étudié. On sait que le sperme de cheval est très dilué par les sécrétions des glandes accessoires; que la réaction alcaline de ces dernières, leur richesse en électrolytes, surtout en chlore, et leur insuffisance en glucides paraissent être la cause principale de la brièveté de la survie (3-4 h. en moyenne) des spermatozoïdes de cette espèce, conservés in vitro. L'activité initiale, très élevée, des spermatozoïdes de cheval est assurée par les processus oxydatifs, pendant lesquels les ressources intérieures des cellules (protéines, lipides) sont surtout utilisées (Milovanow, I940).

Ainsi, dans le métabolisme des spermatozoïdes de cheval, les protéines cellulaires, et probablement celles du plasma séminal (métabolites endogènes), semblent jouer un rôle plus important que dans le métabolisme du sperme de taureau, par exemple, où la fructolyse prédomine. De plus, les protéines du plasma séminal, en général, semblent avoir une importance considérable, comme substances colloïdales protectrices pour les membranes des spermatozoïdes (Hayashr, I945). Grâce à ces substances, le plasma séminal dialysé (partie non-dialysable), ajouté au sperme conservé in vitro, est susceptible de prolonger la survie des spermatozoïdes (MANN, I949).

Bien que la composition chimique du plasma séminal du cheval ne soit pas connue, et quoiqu'on sache que les protéines du plasma du taureau par exemple se distinguent, par leurs propriétés physiques et fonctionnelles, de celles des spermatozoïdes, la composition du sperme entier du cheval peut donner certaines indications pour le plasma lui-même. 


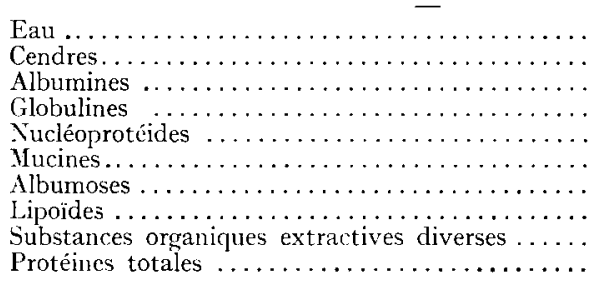

$\left\{\begin{array}{l}95,7 \text { p. I00 } \\ 0,915 \\ 1,442 \\ 0,559 \\ 0,537 \\ 0,172 \\ 1,090 \\ 2,2.38\end{array}\right.$

Il faut ajouter que, d'après Popov (I938), dans le sperme de cheval, l'azote non protéique représente une plus grande partie de l'azote total que dans celui d'autres espèces. Il est probable qu'après l'éjaculation se produit une accumulation de l'azote non protéique, par décomposition très rapide des protéines, sous 1'action des enzymes du sperme (Mann, I954). Selon Sarkar et Coll. (I947), les acides aminés du plasma séminal, à l'exception du tryptophane, de la leucine et de l'arginine, sont presque identiques chez le taureau à ceux des spermatozoïdes.

Les acides aminés et les protéoses, en général, ont une certaine importance pour la conservation de la vitalité des spermatozoïdes. Ils peuvent contrecarrer, au moins en partie, l'action néfaste des électrolytes du sperme. D'après TYLER et Coll. (I95I), cette propriété avantageuse est due au pouvoir des acides aminés de rentrer en liaison chimique avec des métaux. C'est grâce à cette action que l'inclusion des acides aminés (glycine, alanine, valine, leucine, lysine, acide glutaminique) dans les dilueurs augmente la motilité, et, même, la fertilité des spermatozoïdes bovins (MANN, I949).

On peut supposer que le même phénomène, en ce qui concerne l'action des acides aminés, se produit dans le sperme de cheval. Il est peut-être même plus accentué chez cette espèce, la concentration des substances azotées dialysables y étant plus élevée que dans le sperme d'autres espèces.

De plus grandes précisions sur les protéines du plasma séminal ont été obtenues à l'aide de l'électrophorèse. Gray et Coll. (I942) ont trouvé 4 fractions électrophorétiques distinctes des corps protéiniques du plasma séminal humain, dont les motilités électrophorétiques sont à peu près semblables à celles des fractions du sérum sanguin : albumines, globu1lines $x, \beta, \gamma$

Ross et Coll. (I942) ont distingué 5 fractions : $P_{1}$ (Protéoses), $P_{2}$ et $\mathrm{P}_{3}$ (globulines), $\mathrm{P}_{4}$ (glycoprotéines) et $\mathrm{P}_{5}$ (semble provenir de $\mathrm{P}_{4}$ pendant la dialyse). Ils ont rencontré seulement des traces d'albumines et de nucléo-protéines. Ces résultats ont été confirmés par des études sérologiques et spectrophotométriques.

Larson et Coll. (I954) ont distingué, dans le plasma séminal du taureau, II fractions de protéines parmi lesquelles les fractions II, Io et 9 , qui, d'après leur motilité électrophorétique, correspondaient aux albu- 
mines. D'autres fractions, I à 9, dont l'ensemble représentait une grande partie des protéines totales, ont été déterminées par LaRson et Coll. comme se déplaçant avec des motilités analogues aux globulines du sérum sanguin.

Actuellement, l'électrophorèse sur papier des liquides organiques donne des indications très importantes sur l'état physiologique ou pathologique des différents organes. Ross et Coll. (I943) ont trouvé dans les échantillons de sperme humain anormal la présence régulière à un taux élevé de la fraction $P_{5}$, mais preque l'absence de la fraction $\mathrm{P}_{4}$. Il nous semble donc qu'à l'aide de l'électrophorèse du plasma séminal, il serait possible de dépister les troubles dans la fonction des glandes sexuelles accessoires, ou même de tout le système génital. En outre, l'électrophorèse du plasma séminal peut faire connaître quelles fractions électrophorétiques des protéines dans le sperme sont les plus importantes pour la vitalité et la fertilité des spermatozoïdes. Elle peut indiquer également quelles protéines sont à choisir pour la dilution efficace de ce sperme. C'est pourquoi, nous avons entrepris ces études sur les protéines du plasma séminal de cheval, en utilisant la méthode d'électrophorèse sur papier.

\section{MATERIEL ET TECHNIQUE}

Huit échantillons de sperme ont été récoltés de deux étalons de type arabe, 9 échantillons de neuf étalons ardennais, et 2 échantillons ont été prélevés sur l'épididyme de deux chevaux castrés. Le sperme des étalons a été examiné d'après les méthodes habituelles (volume, concentration en spermatozoïdes, motilité, $\mathrm{pH}$, pourcentage de spermatozoïdes morts et anormaux, durée de réduction de bleu de méthylène). Les poids spécifiques du sperme entier et du plasma séminal ont été déterminés d'après la méthode de Phil,ifs et Van Stykf (I950). Le plasma séminal a été obtenu par centrifugation du sperme à 6 ooo tours par minute, pendant Io minutes.

Pour l'étude électrophorétique, nous avons utilisé un appareil construit d'après le modèle décrit par Girassmann et Hannig (I950). Cet appareil se compose de deux cuves et d'un pont démontable en plexiglas ; il permet 1'électrophorèse simultanée sur 8 à Io rubans-filtres (Whatman $\left.\mathrm{n}^{0} \mathrm{I}\right)$ de $4 \mathrm{~cm}$. de large.

Le tampon employé a été celui de Durrum (I950) : une solution de $0,05 n$ de véronal sodique et de 0,0 I $n$ de véronal. Le plasma séminal à la dose de 0,05 à $0,06 \mathrm{cc}$. a été déposé sur l'extrémité cathodique des rubans.

Les tensions appliquées ont varié entre $I 70$ et $I 50$ volts, de manière à donner au champ électrique une intensité de 2,4 à $I, 8$ volts par centimètre. L'intensité du courant a été de 0,9 à $\mathrm{I}, \mathrm{I}$ milliampère par ruban. I'électrophorèse a été exécutée à la température de $I_{5}$ à $20^{\circ} \mathrm{C}$, pendant 
Io heures. L,es parties des rubans-filtres contenant des protéines, colorées par du bleu de bromophénol, ont été évaluées par la photométrie progressive, et, sur la base de cette dernière, des diagrammes d'extinction pour chaque échantillon examiné ont été établis. La détermination des positions relatives sur le ruban de ces fractions a été effectuée par la méthođe proposée par BoGUTH (I953). L'évaluation quantitative (concentration relative en pourcentages) a été faite par planimétrie des fractions.

Afin de faciliter l'interprétation des résultats graphiques obtenus sur le plasma séminal, quelques analyses électrophorétiques du sérum de sang d'étalons arabes ont été exécutées.

\section{RESULTATS}

A la suite de l'examen des différents caractères du sperme des étalons étudiés, nous avons obtenu les chiffres extrêmes suivants :

\begin{tabular}{|c|c|c|}
\hline Caractires du sperme & Étalons arabes & Étalons ardennais \\
\hline $\begin{array}{l}\text { Volume } \ldots \ldots \ldots \ldots \ldots \ldots \ldots \ldots \ldots \\
\text { Concentration }\left(\text { par } \mathrm{mm}^{3}\right) \ldots \ldots \ldots \ldots \ldots \ldots \ldots \ldots\end{array}$ & $\begin{array}{l}\text { I5-50 cc. } \\
50.000 \text { à } 307.000\end{array}$ & $\begin{array}{l}30-70 \mathrm{cc} \\
60.000 \mathrm{a} 535.000\end{array}$ \\
\hline 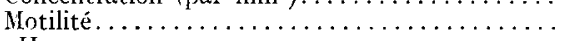 & $30-85$ p. I00 & $70-85$ p. 100 \\
\hline 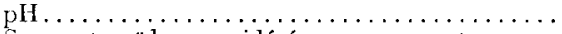 & $6,8-7,1$ & $6,8-7,2$ \\
\hline Spermatozoides considérés comme morts ...... & I3-6I p. I00 & $30-88$ p. 100 \\
\hline Spermatozoides anormaux $\ldots \ldots \ldots \ldots \ldots \ldots$ & $\mathrm{I} 8-32, \mathrm{P}, \mathrm{i} 00$ & $14 ; 36$ p. 100 \\
\hline Temps de réduction du bleu de méthylène ... & $\begin{array}{l}5^{\prime}-\mathrm{I} 5^{\prime} 3 \mathrm{O}^{\prime \prime} \\
\left(\mathrm{T}^{\mathrm{o}}=40^{\circ} \mathrm{C} .\right)\end{array}$ & $\begin{array}{l}\mathrm{IO}^{\prime}-45^{\prime} \\
\left(\mathrm{T}^{\circ}=30^{\circ} \mathrm{C} .\right)\end{array}$ \\
\hline $\begin{array}{l}\text { Poids spécifique du plasma } \ldots \ldots \ldots \ldots \ldots \ldots \\
\text { Poids spécifique du plasma prélevé dans l’épidi- }\end{array}$ & I,OI4-I,OI6 & I,OIO-I,OI8 \\
\hline dyme & 1,038 & \\
\hline
\end{tabular}

Comme le démontre ce tableau, le poids spécifique du plasma d'origine épididymaire est plus élevé que celui du sperme, puisqu'il contient probablement davantage de substances à grosses molécules (protéines).

L'examen électrophorétique du plasma étudié a permis de distinguer des fractions de protéines principales $a, b, c$, correspondant, d'après leur motilité électrophorétique, aux globulines du sérum de sang, et des fractions $\mathrm{P} a_{1-3}$ ayant une motilité qui se rapproche de celles de l'albumine du sérum sanguin.

\begin{tabular}{|c|c|c|c|c|c|}
\hline Plasma d'étalons & $\mathrm{P} a_{1-3}$ & $a_{1}$ & $a_{2}$ & $\mathrm{~B}_{1-4}$ & $c_{1-3}$ \\
\hline Arabes & 2,9 & 17,6 & 16,3 & 30,9 & 32, \\
\hline 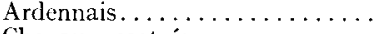 & 3,4 & 20,2 & 17,7 & 31,2 & 27,5 \\
\hline Chevaux castrés $\ldots \ldots \ldots \ldots \ldots$ & 3,8 & I 4,I & 20,0 & 43,9 & 18,2 \\
\hline
\end{tabular}

Les différences entre les concentrations relatives de diverses fractions de protéines du plasma séminal chez les étalons arabes et chez les ardennais se font remarquer surtout dans la fraction $c$; elles semblent être dues plutôt à l'âge qu'à la race ( 2 arabes de II et 26 ans ; 9 ardennais de 3 à 4 ans). En analogie avec I,ARSON et Coll. (I954), la fraction $c$ est supposée contenir les immunoglobulines. 
Le plasma d'origine épididymaire (obtenu après la castration) se caractérise, en comparaison avec celui du sperme d'étalons, par une concentration relativement basse des fractions $a_{1}$ (I4, Ip. Ioo) et $c_{1-3}$ (I 8,2 p. I0o) et par une concentration excessivement haute des fractions $b_{1-4}$ (43,9 p. roo). La concentration des fractions $\mathrm{P}_{1-3}$ du plasma de sperme normal semble être presque égale à celle dı plasma épididymaire.

Comme nous 1'avons déjà signalé, Ross et Co11. (I943) ont trouvé dans les échantillons du sperme humain anormal la présence régulière de la fraction $P_{5}$, ayant une motilité électrophorétique plus grande que d'autres fractions de ce sperme.

Dans le plasma séminal de cheval, la plus grande vitesse de déplacement appartient aux fractions $\mathrm{P}_{1-3}$. On peut donc se demander si, par analogie, une haute concentration de ces fractions dans le plasma de cheval n'indique pas également un état pathologique du sperme chez cette espèce.

Parmi les étalons ardennais étudiés, nous avons observé un sujet dont le plasma séminal a montré une haute concentration moyenne des fractions $\mathrm{P} a_{1-3}$ s'élevant à II,5 p. Ioo. D'autre part, l'examen du sperme de cet étalon a révélé que ses caractères, consignés dans le tableau cidessus, sont loin d'être normalx : motilité : $75 \mathrm{p}$. Ioo ; concentration en spermatozoïdes : 85 ooo $p / \mathrm{mm}^{3} ; \mathrm{pH}: 7,2$; pourcentage des spermatozoides anormaux : $36 \mathrm{p}$. Ioo (1a limite admissible pour le sperme normal est de $15 \mathrm{p} . \mathrm{IOO})$; temps de réduction du bleu de méthylène (à $\left.30^{\circ} \mathrm{C}\right): 45^{\prime}$; poids spécifique : I,orI.

Ainsi, nous appuyant sur ces observations et celles de Ross et Coll. (I943), nous pouvons supposer que, en général, la présence dans le plasma séminal d'un cheval des fractions $\mathrm{P} a_{1-3}$ dans une concentration relative supérieure à Io $\mathrm{p}$. Ioo permet de soupçonner des causes pathologiques de leur formation, par exemple certaines lésions exsudatives dans les organes ou les voies génitales.

Il est évident qu'une connaissance plus complète de l'importance des différentes fractions des protéines du plasma séminal pour la vitalité et la fertilité des spermatozoïdes chez le cheval ou chez d'autres espèces, exige des études approfondies. Cependant, nos observations permettent de se demander si la concentration relativement élevée des fractions $b_{1-4}$ dans le plasma séminal épididymaire a une signification pour la maturation et le métabolisme des spermatozoïdes dans 1'épididyme. On sait que les spermatozoïdes qui y sont prélevés, sont souvent non mobiles, mais que leur survie est très longue. On peut donc supposer que ces fractions de protéines servent comme facteur de longévité de sperme, surtout chez le cheval, où les protéines semblent être les sources nutritives possibles du métabolisme.

Ensuite, en ce qui concerne le rôle physiologique des différentes frac- 
tions des protéines du plasma séminal, nous avons trouvé quelques corrélations significatives : par exemple, entre la concentration des fractions $a_{1}$ d'une part, et la concentration du sperme en spermatozoïdes $(+0,82 \pm 0, \mathrm{I} 2$, chez les étalons arabes), la motilité $(+0,89 \pm 0,08$, arabes), le temps de réduction du bletu de méthylène $(-0,70 \pm 0,19$, arabes) d'autre part ; entre la concentration des fractions $a_{2}$ et la concentration en spermatozoïdes $(+0,52 \pm 0, \mathrm{I} 8$, arabes) ; entre la concentration de l'ensemble des fractions $a, b, c$ et la concentration du sperme en spermatozoïdes $(+0,24 \div 0,09$, pour les étalons arabes et ardennais envisagés ensemble), tandis que la corrélation entre la concentration des fractions $\mathrm{Pa}_{1-3}$ d'une part, et la concentration en spermatozoïdes d'autre part a été négative et non significative (-0,2I $\pm 0, I_{5}$, pour tous les étalons).

Nous rappelons ici ces coefficients de corrélation seulement à titre d'information, puisque nos observations (I8 échantillons de sperme en tout) ne sont pas assez nombreuses pour qu'on puisse titer quelque conclusion précise. Néanmoins, on peut supposer que l'ensemble des fractions des protéines $a_{1}, a_{2}, b_{1-4}, c_{1-3}$ et surtout les fractions $a_{1}$ et $a_{2}$, sont essentielles pour l'activité et la vitalité des spermatozoïdes, tandis qu'une haute concentration des fractions $\mathrm{P}_{1-3}$ du plasma séminal semble être néfaste pour ces propriétés du sperme de cheval.

Une fois connue la valeur physiologique des différentes fractions de protéines dans le métabolisme du sperme et vu le manque dans le plasma des protéines à motilité albuminique, on pourra essayer de trancher la question de leur valeur pour la dilution du sperme.

Tosic et WaLTon (I946) ont constaté que la partie dialysable du jaune d'œuf qui est utilisé normalement pour la dilution du sperme de taureau, contient des substances inhibant la respiration des spermatozoïdes et, par conséquent, diminuant leur pouvoir fécondant. I1 nous semble que les fractions $\mathrm{P} a_{1-3}$ des protéines, introduites dans le dilueur, peuvent avoir une action néfaste comparable. Mais ce dernier phénomène ainsi que l'action d'autres fractions des protéines envisagée séparément, demandent des études spéciales. Thacker (D.) et Coll. (I954) ont déjà mis en évidence la toxicité pour les spermatozoïdes bovins des fractions du sérum de lait non pasteurisé, contenant des albumines. Au contraire, les fractions des globulines de ce lait (euglobulines et pseudo-globulines), sont favorables pour la vitalité des spermatozoïdes de cette espèce.

\section{CONCLUSION}

Le rôle des protéines dans le métabolisme du sperme, en général, est peu connu, mais il semble être non négligeable dans le sperme de cheval (Milovanow, I940), où les protéines sont les sources possibles de l'activité des spermatozoïdes. 
L'électrophorèse sur papier du plasma séminal du cheval montre la présence dans celui-ci des fractions des protéines analogues à celles du sérum de sang: $\mathrm{P} a_{1-3}$ (albumines), $a_{1}, a_{2}, b_{1-4}$ et $c_{1-3}$ (globulines).

L'analyse électrophorétique du plasma séminal semble pouvoir faciliter la détermination de la valeur physiologique du sperme pour la fécondation, ainsi que le dépistage des troubles fonctionnels de l'appareil génita1.

La présence dans le plasma séminal de cheval des fractions $\mathrm{P}_{a_{1-3}}$ en une concentration supérieure à Io p. Ioo paraît caractériser le sperme anormal de cette espèce.

Les études de la valeur de différentes fractions des protéines du plasma séminal peuvent donner des indications pour la préparation des dilueurs de sperme chez le cheval et chez d'autres espèces.

\section{RÉFÉRENCES BIBLIOGRAPHIQUES}

(I) MrLovanow (W.), I940. -- Insémination artificielle des animaux domestiques.

(2) Mann (T.), I949. - Advances in enzymology.

(3) Slovtsow, voir Milovanow, I940.

(4) Popow (M.), voir Milovanow, I940.

(5) MANN (T.), r954. - Biochemistry of semen, London.

(6) Sarkar (B.) et Coll., I947. - J. Biol. Chem., 171, 463.

(7) 'TYler et Coll., I95I, voir MaNn (T.), I954.

(8) Gray (S.) et Coll., I942. - Proc. Soc. Exp. Biol. Med. 50, 35I.

(9) Ross (V.) et Coll., I942. - J. Biol. Chem., 144, 667.

(IO) Larson (B.) et Coll., I954. - J. Biol. Chem., 206, 74I.

(II) Boguth (W.), I953. - Zentrabl. f. Vet. Med., 1, I68.

(I2) Ross et Coll., I943. - Proc. Soc. Exp. Biol. Med., 54, I79.

(i3) Grassmann (W.) et Hannig (K.), I950. - Naturwissenjten, 37, 496.

(I4) Durrum (E.), I950. - J. Amer. Chem. Soc., 72, 2943.

(I5) Phillips (R.) et Van Slyke (D.), I950. - J. Biol. Chem., 183, 305.

(i6) Tosic (J.) et Walton (A.), I946. - J. $A g r . S c ., 37,69$.

(I7) Thacker (D.) et Coll. (I954). - J. Dairy Sci., 37, 220.

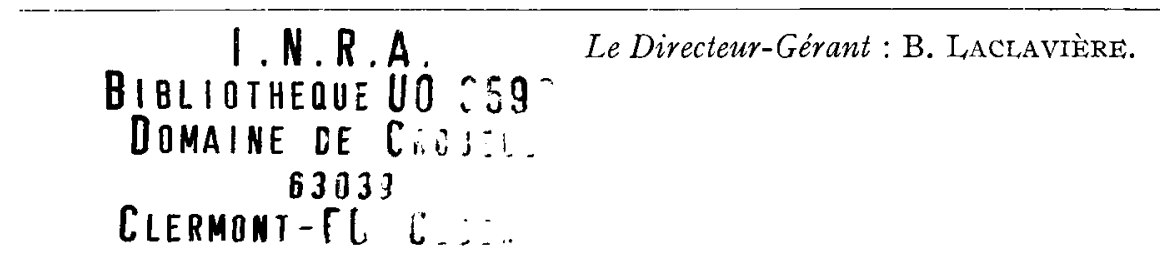

